AIP Applied Physics

\title{
Magnetism, magnetoresistance, and Shubnikov-de Haas oscillations in Na- implanted highly oriented pyrolitic graphite
}

R. F. Pires, P. Pureur, M. Behar, J. L. Pimentel, J. Schaf et al.

Citation: J. Appl. Phys. 111, 093922 (2012); doi: 10.1063/1.4709731

View online: http://dx.doi.org/10.1063/1.4709731

View Table of Contents: http://jap.aip.org/resource/1/JAPIAU/v111/i9

Published by the American Institute of Physics.

\section{Related Articles}

Fabrication of samples for scanning probe experiments on quantum spin Hall effect in $\mathrm{HgTe}$ quantum wells J. Appl. Phys. 112, 103713 (2012)

Tunneling conductance and magnetoresistance in topological insulator $\mathrm{Fi} / \mathrm{I} / \mathrm{Fi} / \mathrm{d}$-wave superconductor junctions J. Appl. Phys. 112, 103919 (2012)

Room temperature magnetoresistance in a polyimide-Co granular film synthesized by vapor deposition polymerization

Appl. Phys. Lett. 101, 222401 (2012)

Magneto-resistance up to 60 Tesla in topological insulator Bi2Te3 thin films

Appl. Phys. Lett. 101, 202403 (2012)

High magnetoresistance in graphene nanoribbon heterojunction

Appl. Phys. Lett. 101, 183111 (2012)

\section{Additional information on J. Appl. Phys.}

Journal Homepage: http://jap.aip.org/

Journal Information: http://jap.aip.org/about/about_the_journal

Top downloads: http://jap.aip.org/features/most_downloaded

Information for Authors: http://jap.aip.org/authors

\section{ADVERTISEMENT}

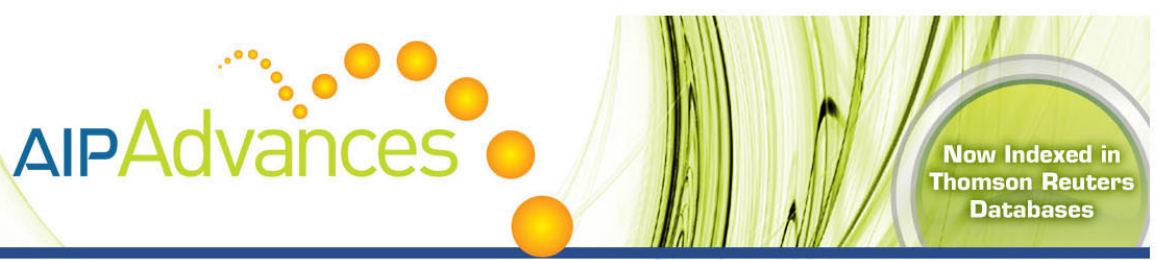

\section{Explore AIP's open access journal: Rapid publication \\ - Article-level metrics \\ Post-publication rating and commenting}




\title{
Magnetism, magnetoresistance, and Shubnikov-de Haas oscillations in Na-implanted highly oriented pyrolitic graphite
}

\author{
R. F. Pires, ${ }^{1}$ P. Pureur, ${ }^{1}$ M. Behar, ${ }^{1}$ J. L. Pimentel, Jr., ${ }^{1}$ J. Schaf, ${ }^{1}$ and Y. Kopelevich ${ }^{2}$ \\ ${ }^{1}$ Instituto de Física, Universidade Federal do Rio Grande do Sul, 91501-970, P.O. Box 150151, Porto Alegre, \\ RS, Brazil \\ ${ }^{2}$ Instituto de Física “Gleb Wathagin,” Universidade Estadual de Campinas, UNICAMP, 13083-970, \\ Campinas, SP, Brazil
}

(Received 19 January 2012; accepted 29 March 2012; published online 11 May 2012)

\begin{abstract}
We report on magnetization, magnetoresistance, and Shubnikov-de Haas oscillations experiments in Na-implanted samples of highly oriented pyrolitic graphite (HOPG). Different ion fluences were applied so that samples with $\mathrm{Na}$ contents of $0.5,1.0,1.5$, and 2.0 at. \% were obtained in the implanted region. Ferromagnetic-like hysteresis was observed in magnetization experiments where the field was applied parallel to the graphene planes. The observed saturation moment increases systematically as a function of the implanted ion concentration up to $\mathrm{Na} 1$ at. \%, where it goes through a maximum before decreasing slightly towards $\mathrm{Na} 2$ at. \%. The planar magnetoresistance amplitude at fixed field and temperature closely correlates with the saturation magnetization data. This result suggests that the strong planar magnetoresistance in graphite is at least partially related to a spin dependent mechanism. The magnetoresistance experiments also reveal the occurrence of Shubnikov-de Haas oscillations. The characteristic frequencies and the effective masses could be estimated and do not depend on the Na concentration. The reported experiments show that the expressive enhancement observed in ferromagnetic-like response in Na-implanted HOPG is primarily due to point defects produced by the implantation process. (C) 2012 American Institute of Physics. [http://dx.doi.org/10.1063/1.4709731]
\end{abstract}

\section{INTRODUCTION}

The observation of ferromagnetic and superconductinglike hysteresis loops in highly oriented pyrolitic graphite $(\mathrm{HOPG})^{1}$ has been an important motivation for the renewed research activity aimed to understand the electronic properties of this system and carbon-based materials. ${ }^{2}$ Specifically concerning the magnetic behavior of graphite, a weak ferromagnetic response has been systematically encountered superimposed to the strong diamagnetic background when the field is applied parallel to the graphene planes. ${ }^{1,3}$ Although showing some sample dependence, the observed ferromagnetic hysteresis cannot be simply attributed to the effect of impurities. ${ }^{3}$ It is now clear that point defects play a dominant role in the mechanism giving origin to ferromagnetism in graphite. ${ }^{4}$ Experiments of proton irradiation ${ }^{4,5}$ and theoretical calculations ${ }^{6,7}$ indicate that vacancies and vacancy-hydrogen complexes may induce magnetic moments in this material. Studies using positron annihilation in carbon ion implanted HOPG (Ref. 8) also show that magnetic moments in this system are closely correlated to the presence of vacancies.

On the other hand, magneto-transport experiments in HOPG and Kish single crystal graphite clearly reveal quantum phenomena such as the Shubnikov-de Haas $(\mathrm{SdH})$ oscillations $^{9-12}$ and the quantum Hall effect. ${ }^{11-13}$ The analysis and interpretation of these results have been related to the role of normal carriers ${ }^{9,11}$ and Dirac fermions that are known to govern the electronic properties of graphene. ${ }^{11,14}$ An interesting comparative analysis of the Berry phase effects between graphite and other two-dimensional electron systems is presented in Ref. 12 .

In the present study, we carried out magnetic moment measurements in conjunction with magnetoresistance experiments in HOPG samples implanted with $\mathrm{Na}$ ions at different fluences. Ferromagnetic-like hystereses were systematically observed when the field was applied parallel to the graphene layers. It is shown that the saturation moment is a nonmonotonic function of the implanted $\mathrm{Na}$ concentration and correlates with the planar magnetoresistance measured at fixed temperatures. Moreover, these experiments reveal the occurrence of SdH oscillations. The characteristic frequencies could be estimated and do not depend on the Na concentration. The oscillation amplitude could be qualitatively analysed using the Lifshitz-Kosevich theory. ${ }^{15}$ Our study aims to investigate the effects of $\mathrm{Na}$ ion implantation on the ferromagnetic response of HOPG, as well as to check for the possibility of $\mathrm{Na}$ doping in the graphene atomic planes of HOPG. Our findings are discussed in view of the effects produced by $\mathrm{H}$ and $\mathrm{He}$ irradiation ${ }^{4,5}$ as well as on observations obtained from carbon ion implantation ${ }^{8}$ in this system.

\section{EXPERIMENTAL}

Four HOPG samples were cut out of a pellet sourced by SPI supplies (SPI-1 grade). ${ }^{16}$ The samples typically have dimensions $5 \times 5 \times 0.25 \mathrm{~mm}$. Before any further processing, some of these samples were submitted to particle induced $\mathrm{x}$-ray emission (PIXE) analysis. Impurities at the level of $12 \mathrm{ppm} \mathrm{Fe}$ and $5 \mathrm{ppm} \mathrm{Ca}$ were detected. One of the four 
samples was left pristine. The other four samples were submitted to $\mathrm{Na}$ implantation on one of their large faces. The implantations were carried out at the $500 \mathrm{keV}$ Ion Implanter at the Instituto de Física, Porto Alegre. The fluences were $0.5,1,1.5$, and $2 \times 10^{16} \mathrm{Na} / \mathrm{cm}^{2}$ implanted in samples labelled GNa-0.5, GNa-1, GNa-1.5, and GNa-2, respectively. In all cases, according to the TRIM code,${ }^{17}$ the distribution of the implanted atoms has a Gaussian profile with center $180 \mathrm{~nm}$ below the irradiated surface and $40 \mathrm{~nm}$ half-width. Concentrations of $0.5,1,1.5$, and 2 at. \% Na were obtained near the peak of the Gaussian distribution for samples GNa0.5 , GNa-1, GNa-1.5, and GNa-2, respectively. Table I lists the relevant implantation parameters used in this study.

Magnetic measurements were performed in all the five studied samples with a Quantum Design MPMS@ SQUID magnetometer. ${ }^{18}$ For each sample, the magnetic moment was measured in the applied field $\mu_{0} H=0.01 \mathrm{~T}$ as a function of temperature between $5 \mathrm{~K}$ and $300 \mathrm{~K}$. Hysteresis loops were collected at $T=30 \mathrm{~K}$ in the field range between $-2 \mathrm{~T}$ and $+2 \mathrm{~T}$. The field was oriented either parallel or perpendicular to the $c$-axis in all magnetic experiments.

Magnetoresistance experiments were performed in all the prepared HOPG samples. However, the investigation of the quantum oscillation effects was limited to the virgin sample (labelled G-pure), and the GNa-1 and GNa-2 samples. These experiments were carried out in a Quantum Design PPMS@ system. ${ }^{18}$ Four in-line electrical contacts were attached with silver paint to one of the large surfaces of the studied samples. In the case of the Na-implanted samples, the contacts were attached to the irradiated face. The magnetoresistence was measured in the fixed temperatures $T=2 \mathrm{~K}$, $5 \mathrm{~K}$, and $15 \mathrm{~K}$ as a function of the magnetic field that was changed in the range between $-9 \mathrm{~T}$ and $+9 \mathrm{~T}$. In these experiments, the magnetic field was kept aligned to the $c$-axis.

\section{RESULTS}

Representative results for the anisotropic magnetization of our samples are shown in Fig. 1. Measurements in the non-irradiated G-pure sample are shown in fields between $-2 \mathrm{~T}$ and $+2 \mathrm{~T}$ for the fixed temperature $T=30 \mathrm{~K}$. The axial magnetization $M_{c}$ obtained when the field is applied parallel to the $c$-axis is depicted in Fig. 1(a). The dc susceptibility calculated from these data is $\chi_{c}=-1.8 \times 10^{-3}$ (SI)

TABLE I. Parameters for Na implantation in HOPG.

\begin{tabular}{lccc}
\hline \hline & \multicolumn{3}{c}{ Samples } \\
\cline { 2 - 4 } Parameters & $\mathrm{G}(\mathrm{Na})-0.5$ & $\mathrm{G}(\mathrm{Na})-1$ & $\mathrm{G}(\mathrm{Na})-2$ \\
\hline Energy $(\mathrm{KeV})$ & 120 & 120 & 120 \\
Current $\left(\mathrm{nA} / \mathrm{cm}^{2}\right)$ & 50 & 50 & 80 \\
Temperature $\left({ }^{\circ} \mathrm{C}\right)$ & 30 & 30 & 30 \\
Doses $\left(10^{16} \mathrm{~cm}^{2}\right)$ & 0.5 & 1 & 2 \\
At. $\% \mathrm{Na}^{\mathrm{a}}$ & 0.5 & 1 & 2 \\
Depth $(\mathrm{nm})^{\mathrm{b}}$ & 180 & 180 & 180 \\
Half-width $(\mathrm{nm})$ & 40 & 40 & 40 \\
\hline \hline
\end{tabular}

${ }^{\mathrm{a}}$ At the maximum of the implanted distribution.

${ }^{\mathrm{b}}$ Gaussian profile.

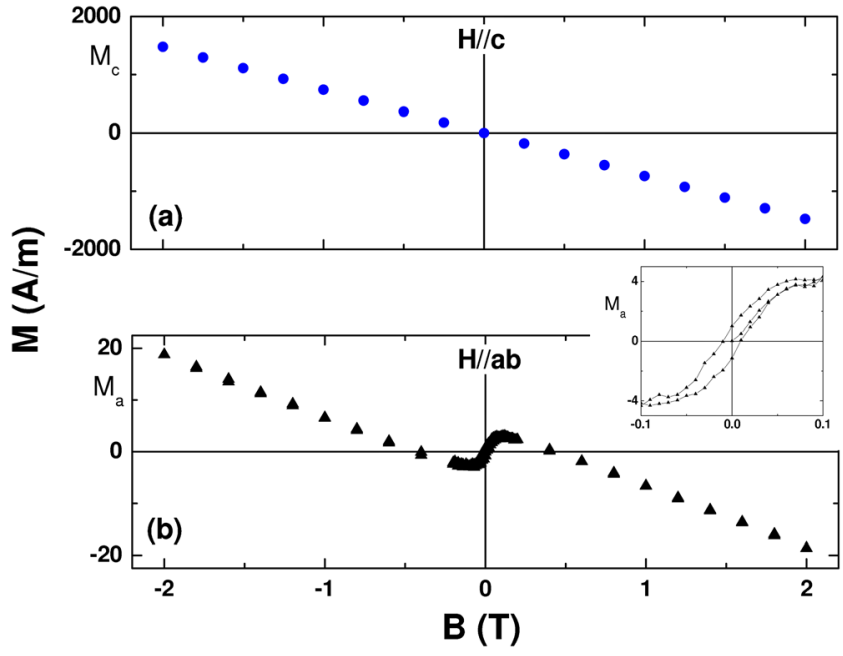

FIG. 1. Magnetization of the virgin HOPG sample (G-pure) at $T=30 \mathrm{~K}$ in fields applied (a) parallel and (b) perpendicular to the c-axis. The inset magnifies the ferromagnetic-like hysteresis cycle observed in the low-field magnetization perpendicular to the c-axis.

(or $\chi_{c}=-3.3 \times 10^{-5} \mathrm{emu} / \mathrm{g}$ ), which is accordance with values reported in the litterature. ${ }^{3}$ Figure 1(b) shows the planar magnetization $M_{a}$. The ratio between axial and planar diamagnetic susceptibilities is $\chi_{c} / \chi_{a} \simeq 64$ at $T=30 \mathrm{~K}$ for Gpure. Our HOPG has a rocking curve FWHM near $2^{\circ}$. The susceptibility anisotropy can be much larger in samples having smaller grain misalignment. In the implanted samples, the susceptibility anisotropy decreases in proportion to the $\mathrm{Na}$ concentration, so that $\chi_{c} / \chi_{a} \simeq 47$ in GNa-2. One clearly discerns the small ferromagnetic-like contribution to the bulk magnetization $M_{a}$ around $B=0$, as magnified in the inset of Fig. 1.

Figure 2 shows the planar magnetization loops for all samples in the field range $-2 \mathrm{~T} \leq B \leq 2 \mathrm{~T}$. These cycles were obtained from the $M_{a}$ measurements after discounting the diamagnetic background. The shape of the inner loop as well as the value of the coercive field do not vary appreciably along the series. However, the saturation magnetization $M_{a}^{\text {sat }}$ varies systematically with the Na content, as depicted in Fig. 3. One observes in this figure that $M_{a}^{\text {sat }}$ increases with the implanted ion content up to 1 at. $\% \mathrm{Na}$, reaches a maximum around this concentration, then decreases slightly for samples with higher Na content. The implantation effect is quite expressive since $M_{a}^{\text {sat }}$ in GNa-1 increases up to $40 \%$ with respect to the value measured for the virgin sample.

Figure 4 shows representative results for the planar magnetoresistance as a function of the field applied parallel to the $c$-axis. A schematic view of the geometry adopted in our magnetoresistance experiments is shown in the inset of Fig. 4. Since the four electrical contacts are placed on the implanted face, the current is expected to flow mainly close this surface owing to the enormous resistivity anisotropy of graphite. Thus, in spite of the fact that implantation modifies only a thin superficial layer of our samples, we expect that the magnetoresistance experiments are indeed probing the effects of irradiating HOPG with $\mathrm{Na}$ ions. The temperature was kept fixed in $T=2 \mathrm{~K}$ and the field was swept between $-9 \mathrm{~T}$ and $+9 \mathrm{~T}$. Similar experiments were performed in 


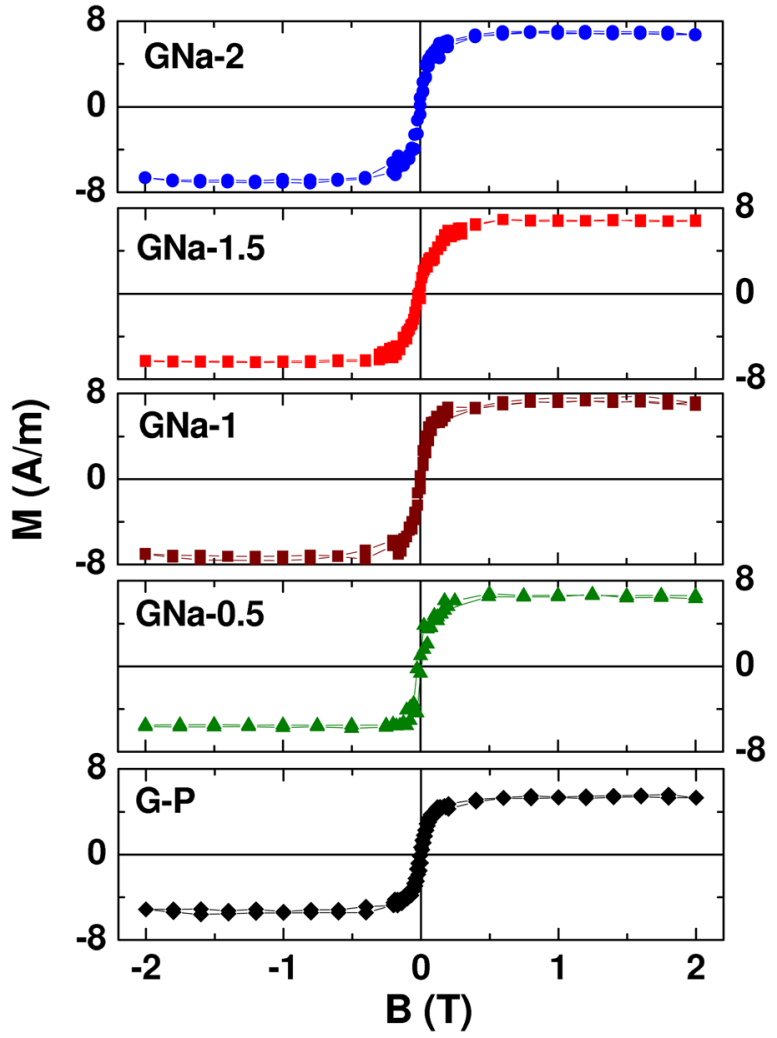

FIG. 2. Hysteresis cycles measured in fields varying between $-2 \mathrm{~T}$ and $+2 \mathrm{~T}$ when applied perpendicular to the $c$-axis of the studied Na implanted HOPG samples. The diamagnetic background is subtracted.

temperatures $T=5 \mathrm{~K}, 15 \mathrm{~K}$, and $50 \mathrm{~K}$. In Fig. 4, the planar magnetoresistance is plotted as

$$
\frac{\Delta \rho_{a}}{\rho_{a}}=\frac{\rho_{a}(B)-\rho_{a}(0)}{\rho_{a}(0)} .
$$

The field induced resistivity variation is very large, much bigger than the giant magnetoresistance observed in magnetic multilayers, ${ }^{19}$ and can be comparable with the collossal magnetoresistance observed in some manganites. ${ }^{20}$ The magnetoresistance for the GNa-1 sample is the largest among our samples and amounts to nearly $1000 \%$ at $B=5$

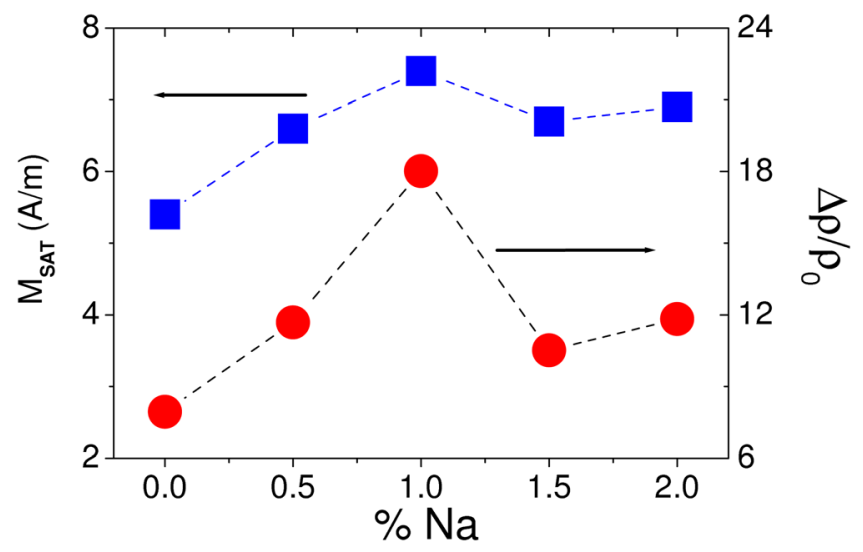

FIG. 3. Saturation magnetization at $T=30 \mathrm{~K}$ (represented as squares) of the ferromagnetic-like response for the pure and Na-implanted HOPG samples and the corresponding planar magnetoresistance magnitudes (circles) measured in $\mu_{0} H=5 \mathrm{~T}$ at the same temperature.

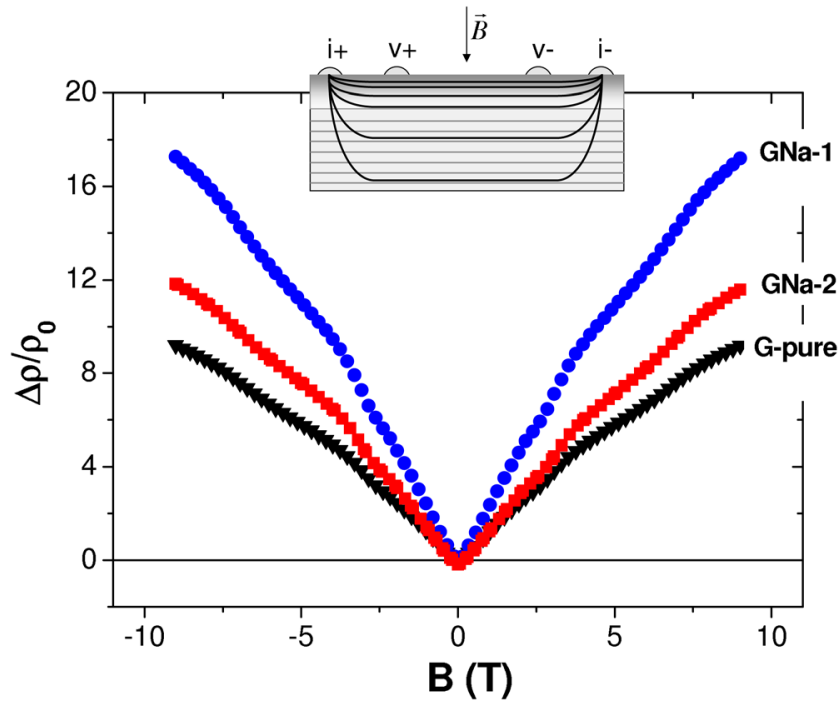

FIG. 4. Magnetoresistance perpendicular to the $c$-axis as a function of the applied field for the quoted samples. The measurements were performed at $T$ $=2 \mathrm{~K}$. The inset shows the configuration adopted for attaching the electrical contacts and schematizes the expected current flow.

T. In Fig. 3, we plot $\Delta \rho_{a} / \rho_{a}$ measured at $B=5 \mathrm{~T}$ and $T=2$ $\mathrm{K}$ as a function of the Na content. A remarkable correlation of the magnetoresistance amplitude with the $M_{a}^{\text {sat }}$ results is observed. This correlation remains valid independently of the field and temperature values used to measure $\Delta \rho_{a} / \rho_{a}$.

The occurrence of Shubnikov-de Haas oscillations are discernible in the high field range of results in Fig. 4. In order to magnify these quantum oscillations, we fit smooth polynomials of the type $r(B)=a B-b B^{2}$ to each magnetoresistance curve. We then subtract this average curve from the measured data as

$$
\delta \rho_{\text {osc }}=\frac{\Delta \rho_{a}(B)}{\rho_{a}(0)}-r(B) .
$$

In Fig. 5, we plot $\delta \rho_{\text {osc }}$ for G-pure in temperatures $T=$ 2,5 , and $15 \mathrm{~K}$. Similar analyses were done for GNa-1 and

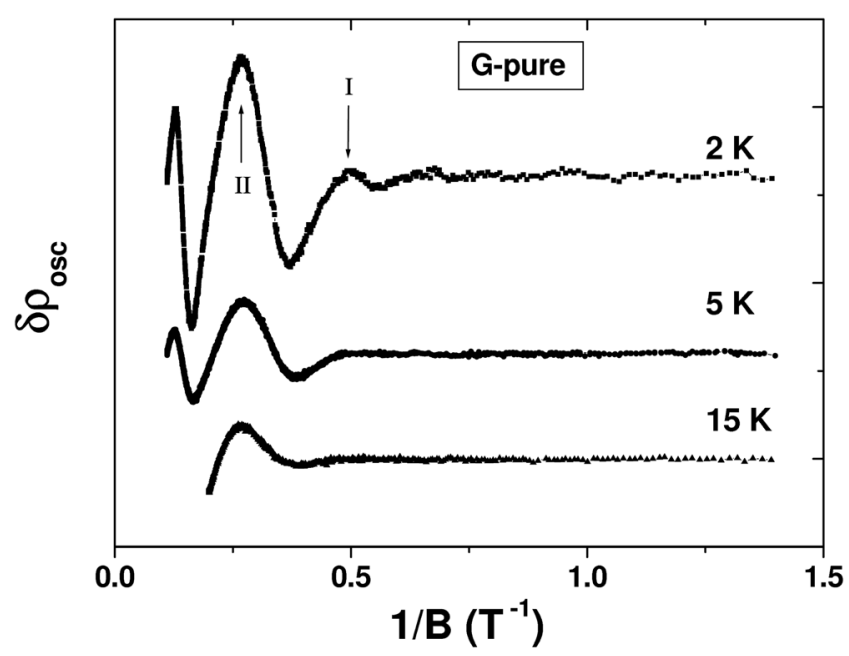

FIG. 5. Shubnikov-de Haas oscillations in the magnetoresistance of the virgin HOPG sample at the quoted temperatures. The temperature dependence of the oscillation amplitudes labelled as I and II were analysed with Eq. (3) for samples GNa-1 and GNa-2. 


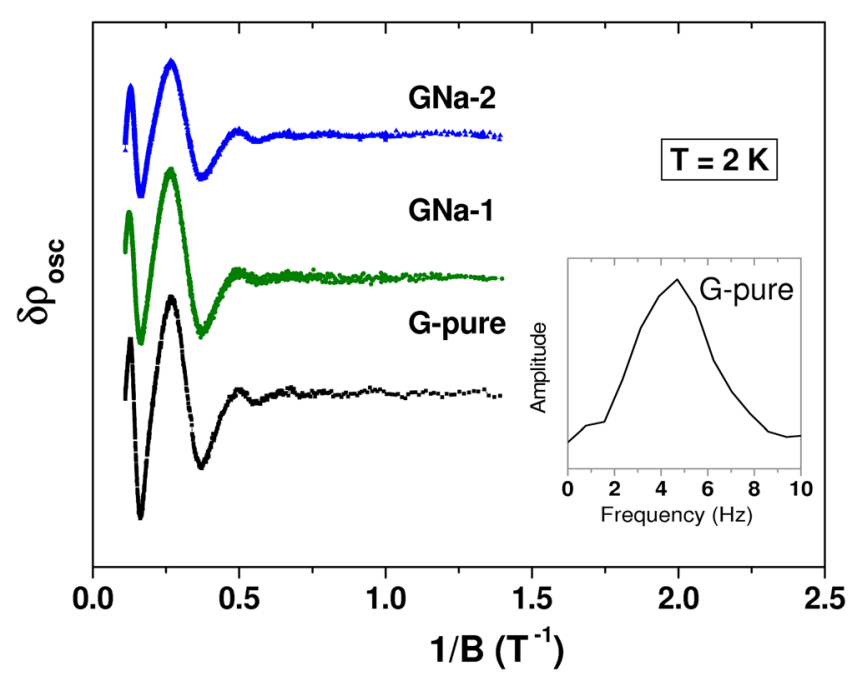

FIG. 6. Shubnikov-de Haas oscillations in the magnetoresistance of the virgin and $\mathrm{Na}$ implanted GNa-1 and GNa-2 HOPG samples at $T=2 \mathrm{~K}$. The inset shows the amplitude of the fast Fourier transform applied to results for the G-pure sample.

GNa-2. Figure 6 displays the oscillations observed in $T=2$ $\mathrm{K}$ for these samples. In $T=50 \mathrm{~K}$, we could not discern any $\mathrm{SdH}$ oscillations in the magnetoresistance measurements.

\section{DISCUSSION}

The measured saturation magnetization in our samples is rather small. For the virgin sample, $M_{\text {sat }}=5.2 \mathrm{~A} / \mathrm{m}$. This corresponds to a magnetic moment $\mu_{C}=5.0 \times 10^{-6} \mu_{B}$ per carbon atom. Owing to the $12 \mathrm{ppm}$ of Fe detected into our HOPG samples, this moment could be explained if one admits that about $20 \%$ of these impurities segregate into clusters where the bulk Fe moment is preserved. However, segregation of $\mathrm{Fe}$ in graphite has not been seen experimentally. $^{21}$ On the other hand, several experimental reports $^{1,3,5,8,22}$ as well as theoretical calculations ${ }^{6,7,23}$ claim that the ferromagnetic-like moments in graphite have origin in point lattice defects. Vacancies and vacancy complexes are supposed to be the more efficient defects to generate moments in graphene and graphite. ${ }^{7,8,23}$ Indeed, the systematic enhancement of $M_{\text {sat }}$ due to $\mathrm{Na}$ ion implantation indicates that point defects of the type produced by ion irradiation are at least partially responsible for the ferromagnetic response in HOPG. We notice that the implantation induced augmentation of $M_{\text {sat }}$ is a substantial effect since just a thin superficial layer of the samples is affected. For instance, for $\mathrm{G}(\mathrm{Na})-1$, the enhancement is $\delta M_{\text {sat }} \simeq 2.1 \mathrm{~A} / \mathrm{m}$. Normalizing this value with respect to the irradiated volume (depth $200 \mathrm{~nm}$ ), we estimate that the carbon moment induced by implantation is $\delta \mu_{C} \simeq 0.006 \mu_{B}$. This value may be still larger if the effective volume to be considered is the one where the implanted impurities are located.

It is interesting to note in Fig. 3 that $M_{\text {sat }}$ reaches a maximum around 1 at. $\% \mathrm{Na}$, then decreases for higher concentrations. This effect is probably related to the production of vacancy clusters at higher fluences that should be less efficient to generate moments in graphite. Results in Fig. 3 are in qualitative agreement with experiments and calculations reported by authors in Ref. 8, who stressed the role of vacancies and vacancy complexes produced by carbon ion implantation in the ferromagnetic response in HOPG. Our results are also in accordance with expectations from theoretical analyses $^{6,7,23}$ that ascribe ferromagnetism in graphene and graphite to spin polarization of $\pi$ electrons around point defects such as vacancies or chemically bounded impurities. We notice that the observation concerning the saturation magnetization in Fig. 3 resembles very much the effect of $\mathrm{H}$ and He irradiation in graphite recently reported in Ref. 24. On the other hand, the striking correlation observed in Fig. 3 between the amplitude of $\Delta \rho_{a} / \rho_{a}$ measured at fixed field and temperature with the $M_{a}^{\text {sat }}$ results suggests that, at least in part, the planar magnetoresistance in HOPG is related to a spin dependent scattering mechanism. This is a relevant finding since the origin of the huge planar magnetoresistance in graphite remains under discussion. ${ }^{10}$

In order to verify if a chemical functionalization of $\mathrm{Na}$ occurs so that the electronic structure of HOPG is modified, we perform an analysis of the $\mathrm{SdH}$ oscillations in our samples. From the positions of crests and valleys of oscillations in results as those of Figs. 5 and 6, we guess that two $\mathrm{SdH}$ frequencies with values $f_{\text {low }}=4.8(3) \mathrm{T}$ and $f_{\text {high }}=6.7(3) \mathrm{T}$ are present. However, from fast Fourier transform analyses available in popular commercial softwares, we could only clearly discern the lowest frequency with value $f_{\text {low }}=4.7(2)$ $\mathrm{T}$, as exemplified for the G-pure sample in the inset of Fig. 6. This value is independent of the $\mathrm{Na}$ content. In spite of the crudeness of our analyses and the narrow field range where the $\mathrm{SdH}$ oscillations are observed, the lowest frequency is in good agreement with the de Haas-van Alphen period $P_{\text {low }}=0.208 \mathrm{~T}$, reported in Ref. 25 , and the value $f_{\text {low }}=4.7 \mathrm{~T}$ found in the $\mathrm{SdH}$ and quantum Hall effect experiments of Ref. 11. The Shubnikov-de Haas effect was carefully and thoroughly studied in single crystal graphite by Soule et al. ${ }^{9}$ These experiments aimed to the determination of the Fermi surfaces of graphite, so that fields up to $24 \mathrm{~T}$ were applied along many different orientations with respect to the $c$-axis. The lowest frequency extracted from our measurements matches the corresponding $\mathrm{SdH}$ period reported in Ref. 9 for the $H \| c$ orientation.

The lowest frequency $f_{l o w}$ is identified to electron-like orbits ${ }^{9}$ According to band calculations of graphite, ${ }^{26}$ electron and hole-like small pockets are aligned successively along the vertical HKH edges of the hexagonal Brillouin zone. According to magneto-transport experiments, the electron pocket is located around the central point $\mathrm{K}^{27}$ The extremal cross-section areas of the electron and hole Fermi surfaces on the plane perpendicular to the $c$-axis may be estimated from $f(1 / B)=2 \pi e / h A{ }^{28}$ From our results, we estimate $A_{e}=4.6 \times 10^{16} \mathrm{~m}^{-2}$ for carriers assigned to the lowest $\mathrm{SdH}$ frequency. Since this value is independent of the Na concentration within the accuracy of our experiments, we conclude that the implanted $\mathrm{Na}$ ions do not modify appreciably the graphite band structure in the orientations perpendicular to the $c$-axis. This finding suggests that the irradiated $\mathrm{Na}$ ions might kick some carbon atoms off their lattice sites but do not substitute them in HOPG. 
We push our SdH analysis further by fitting the temperature dependence of the oscillations amplitude with the Lifshitz-Kosevich factor, ${ }^{15}$

$$
F_{L K}=\frac{\Gamma T}{\sinh (\Gamma T)},
$$

where $\Gamma=2 \pi^{2} k_{B} / \hbar \omega_{c}$. The cyclotron frequency is given by $\omega_{c}=e B / m^{*}$, where $m^{*}$ is the carrier effective mass. A qualitative analysis could be done for oscillations that are labelled as I and II in Fig. 5. The corresponding oscillations were also studied in the GNa-1 and GNa-2 implanted samples. The amplitude of each oscillation was estimated from averaging the vertical distance between the labelled crests and the adjacent valleys. Figure 7 illustrates the fit obtained for the temperature dependence of oscillation II to the Lifshitz-Kosevich factor for the GNa-1 sample. Similar fits were obtained for both oscillations I and II in the two other samples where the $\mathrm{SdH}$ effect was studied. Table II lists the cyclotron frequencies and effective masses obtained from these analyses.

The effective mass deduced from oscillation II, $m_{I V}^{*}$ $=0.041(2) m_{e}$, is close to that identified to electron-like carriers in Ref. 9, where the value $m^{*}=0.039(1) m_{e}$ is reported. The carriers having effective mass $m_{I I I}^{*}=0.033(2) m_{e}$, identified from the low-field oscillation I, are not reported in Ref. 9. However, a recent experimental study of the field induced splitting of the HOPG electronic Raman spectrum into Landau levels ${ }^{29}$ leads to an effective mass whose value is exactly the same as that we have found for $m_{I I I}^{*}$. Authors in Ref. 29 attribute this effective mass to chiral fermions with Berry phase $2 \pi$, also seen in graphene bilayers. ${ }^{30}$

In the case of our study, the relevant point is that the $\mathrm{Na}$ implantation does not modify the effective masses identified from fitting the Lifshitz-Kosevich factor to the temperature dependence of the SdH amplitudes in HOPG. This finding further confirms that the implanted $\mathrm{Na}$ ions do not produce significant doping in graphite. Probably, the $\mathrm{Na}$ ions do not substitute the carbon atoms in this solid and occupy intercalation positions between graphene planes. The significant increase of the ferromagnetic saturation moment resulting

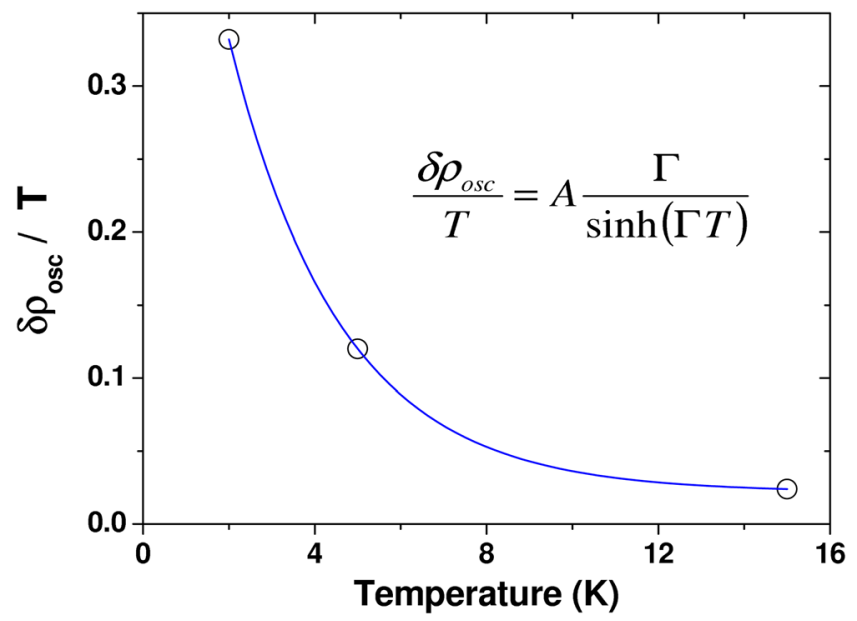

FIG. 7. Fitting of the Lifshitz-Kosevich factor (see Eq. (3)) to the temperature dependent amplitude of the oscillation denoted as II in Fig. 5, but for the GNa-1 sample.
TABLE II. Cyclotron frequencies and effective masses corresponding to oscillations III and IV in the $\mathrm{SdH}$ spectra for the samples G-pure, G(Na)-1, and $\mathrm{G}(\mathrm{Na})-1$.

\begin{tabular}{lccc}
\hline \hline Oscillation & Sample & $\omega_{c}\left(10^{13} \mathrm{~s}^{-1}\right)$ & $m^{*} / m_{e}$ \\
\hline III & G-pure & 1.00 & 0.035 \\
& G(Na)-1 & 1.13 & 0.031 \\
& G-(Na)-2 & 1.08 & 0.033 \\
& Average & & $0.033(2)$ \\
IV & G-pure & 1.62 & 0.040 \\
& G(Na)-1 & 1.62 & 0.040 \\
& G(Na)-2 & 1.53 & 0.043 \\
& Average & & $0.041(2)$ \\
\hline \hline
\end{tabular}

from the Na implantation must then be attributed to site disorder produced in this process.

\section{CONCLUSIONS}

We carried out magnetization and planar magnetoresistance measurement in highly oriented pyrolitic graphite samples implanted with Na. From the magnetic measurements performed with field applied perpendicular to the c-axis, we were able to extract the ferromagnetic-like contribution to the magnetic response of graphite. We showed that the ferromagnetic saturation moment varies as a function of the $\mathrm{Na}$ implantation fluence. This moment increases significantly with the impurity content up to 1 at. \% $\mathrm{Na}$ at the implanted region, reaches a maximum around this concentration, then decreases slightly upon further implantation. The amplitude of the planar magnetoresistance shows a strong correlation with the ferromagnetic moment along the series of Na-implanted HOPG samples, suggesting that a spin dependent scattering mechanism have to be considered to explain the magneto-transport properties of graphite.

Shubnikov-de Haas oscillations are seen in the planar magnetoresistance when the experiments are performed with field applied in the orientation perpendicular to the graphene planes. The characteristic $\mathrm{SdH}$ periods as well the effective masses deduced from the temperature dependence of the oscillation amplitudes do not depend on the implanted Na concentration. This result indicates that $\mathrm{Na}$ does not substitute the carbon atoms and the graphite intrinsic electronic properties remain largely unaffected by implantation, at least in the orientation of the graphene planes.

The magnetoresistance results lead us to conclude that the expressive enhancement of the ferromagnetic-like response in Na-irradiated graphite is primarily due to defects produced by the implantation process. Likely, point defects as isolated vacancies or small vacancy complexes are the most effective to generate magnetic moments since the enhancement of the saturation magnetization goes through a maximum at low implantation fluences. As a final remark, our results show that a significative enhancement of the ferromagnetic response in graphite may be induced by ionic implantation.

\section{ACKNOWLEDGMENTS}

This work is supported in part by CNPq, FAPERGS, and FAPESP, Brazil. 
${ }^{1}$ Y. Kopelevich, P. Esquinazi, J. H. S. Torres, and S. Moehlecke, J. Low Temp. Phys. 119, 691 (2000).

${ }^{2}$ Y. Kopelevich and P. Esquinazi, Adv. Mater. 19, 4559 (2007).

${ }^{3}$ P. Esquinazi, A. Setzer, R. Hohne, C. Semmelhack, Y. Kopelevich, D. Spemann, T. Butz, B. Kohlstrunck, and M. Losche, Phys. Rev. B 66, 024429 (2002).

${ }^{4}$ K.-H. Han, D. Spemann, P. Esquinazi, R. Hhne, V. Riede, and T. Butz, Adv. Mater. 15, 1719 (2003).

${ }^{5}$ P. Esquinazi, D. Spemann, R. Hohne, A. Setzer, K.-H. Han, and T. Butz, Phys. Rev. Lett. 91, 227201 (2003).

${ }^{6} \mathrm{P}$. O. Lehtinen, A. S. Foster, Y. Ma, A. V. Krasheninnikov, and R. M. Nieminen, Phys. Rev. Lett. 93, 187202 (2004).

${ }^{7}$ O. V. Yazyev, Phys. Rev. Lett. 101, 037203 (2008).

${ }^{8}$ X. Yang, H. Xia, X. Qin, W. Li, Y. Dai, X. Liu, M. Zhao, Y. Xia, S. Yan, and B. Wang, Carbon 47, 1399 (2009).

${ }^{9}$ D. E. Soule, J. W. McClure, and L. B. Smith, Phys. Rev. 134, A453 (1964).

${ }^{10}$ Y. Kaburagi, J. Phys. C: Solid State Phys. 15, 5425 (1982).

${ }^{11}$ I. A. Luk'yanchuk and Y. Kopelevich, Phys. Rev. Lett. 93, 166402 (2004).

${ }^{12}$ A. N. Ramanayaka and R. G. Mani, Phys. Rev. B 82, 165327 (2010).

${ }^{13}$ Y. Kopelevich, J. H. S. Torres, R. R. da Silva, F. Mrowka, H. Kempa, and P. Esquinazi, Phys. Rev. Lett. 90, 156402 (2003).

${ }^{14}$ S. Y. Zhou, G.-H. Gweon, J. Graf, A. V. Fedorov, C. D. Spataru, R. D. Diehl, Y. Kopelevich, D.-H. Lee, S. G. Louie, and A. Lanzara, Nat. Phys. 2, 595 (2006)

${ }^{15}$ I. M. Lifshitz and A. M. Kosevich, Zh. Eksp. Teor. Fiz. 29, 730 (1955) [Sov. Phys. JETP 2, 636 (1956)].
${ }^{16}$ See http://www.2spi.com/catalog/new/hopgsub.php for technical information on the studied HOPG.

${ }^{17}$ The Stopping and Range of Ions in Matter, edited by J. F. Ziegler (Pergamon, New York, 1977), Vol. 2-6.

${ }^{18} \mathrm{See} h \mathrm{htp}: / /$ www.qdusa.com/ for general information on the Quantum Design equipments.

${ }^{19}$ M. N. Baibich, J. M. Broto, A. Fert, F. Nguyen van Dau, F. Petroff, P. Eitenne, G. Creuzet, A. Friederich, and J. Chazelas, Phys. Rev. Lett. 61, 2472 (1988).

${ }^{20}$ S. Jin, T. H. Tiefel, M. McCormack, R. A. Fastnacht, R. Ramesh, and L. H. Chen, Science 264, 413 (1994).

${ }^{21}$ R. Hhne, P. Esquinazi, V. Heera, H. Weishart, A. Setzer, and D. Spemann, J. Magn. Magn. Mater. 320, 966 (2008).

${ }^{22}$ H. Ohldag, T. Tyliszczak, R. Hhne, D. Spemann, P. Esquinazi, M. Ungureanu, and T. Butz, Phys. Rev. Lett 98, 187204 (2007).

${ }^{23}$ O. V. Yazyev and L. Helm, Phys. Rev. B 75, 125408 (2007).

${ }^{24}$ T. L. Makarova, A. L. Shelankov, I. T. Serenkov, and V. I. Sakharov, Phys. Status Solidi B 247, 2988 (2010).

${ }^{25}$ S. J. Williamson, S. Foner, and M. S. Dresselhaus, Phys. Rev. 140, A1429 (1965).

${ }^{26}$ J. W. McClure, Phys. Rev. 108, 612 (1957).

${ }^{27}$ J. A. Woolllam, Phys. Rev. B 3, 1148 (1971).

${ }^{28}$ J. M. Ziman, Principles of the Theory of Solids, 2nd ed. (Cambridge University Press, Cambridge, 1979).

${ }^{29}$ A. F. Garcia-Flores, H. Terashita, E. Granado, and Y. Kopelevich, Phys. Rev. B 79, 113105 (2009).

${ }^{30} \mathrm{G}$. Li and Y. Andrei, Nat. Phys. 3, 623 (2007). 